\title{
Network-Based Methods for Identifying Key Active Proteins in the Extracellular Electron Transfer Process in Shewanella oneidensis MR-1
}

\author{
Dewu Ding ${ }^{1,2}$ (1) and Xiao Sun ${ }^{1, *}$ \\ 1 State Key Laboratory of Bioelectronics, School of Biological Science and Medical Engineering, Southeast \\ University, Nanjing 210096, China; dwding2008@aliyun.com \\ 2 Department of Mathematics and Computer Science, Chizhou College, Chizhou 247000, China \\ * Correspondence: xsun@seu.edu.cn; Tel.: +86-139-5198-9906; Fax: +86-25-8379-2349
}

Received: 13 December 2017; Accepted: 12 January 2018; Published: 16 January 2018

\begin{abstract}
Shewanella oneidensis MR-1 can transfer electrons from the intracellular environment to the extracellular space of the cells to reduce the extracellular insoluble electron acceptors (Extracellular Electron Transfer, EET). Benefiting from this EET capability, Shewanella has been widely used in different areas, such as energy production, wastewater treatment, and bioremediation. Genome-wide proteomics data was used to determine the active proteins involved in activating the EET process. We identified 1012 proteins with decreased expression and 811 proteins with increased expression when the EET process changed from inactivation to activation. We then networked these proteins to construct the active protein networks, and identified the top 20 key active proteins by network centralization analysis, including metabolism- and energy-related proteins, signal and transcriptional regulatory proteins, translation-related proteins, and the EET-related proteins. We also constructed the integrated protein interaction and transcriptional regulatory networks for the active proteins, then found three exclusive active network motifs involved in activating the EET process-Bi-feedforward Loop, Regulatory Cascade with a Feedback, and Feedback with a Protein-Protein Interaction (PPI) - and identified the active proteins involved in these motifs. Both enrichment analysis and comparative analysis to the whole-genome data implicated the multiheme c-type cytochromes and multiple signal processing proteins involved in the process. Furthermore, the interactions of these motif-guided active proteins and the involved functional modules were discussed. Collectively, by using network-based methods, this work reported a proteome-wide search for the key active proteins that potentially activate the EET process.
\end{abstract}

Keywords: active protein; extracellular electron transfer; network-based methods; protein-protein interaction; transcriptional regulatory interaction

\section{Introduction}

Shewanella oneidensis MR-1 is one of the most well-known electricigens, which can transfer the electrons produced inside of the cells to the outside of the cells to restore extracellular insoluble solid electron acceptors (extracellular electron transfer, EET) [1,2]. Due to the benefits from this EET capability, there is significant interest in using S. oneidensis MR-1, ranging from energy production and wastewater treatment to bioremediation and biosensing [2-4]. Studying the mechanism of EET is, therefore, a key part in the development of these electricigen-based applications. Generally speaking, S. oneidensis MR-1 can extend its outer membrane to form electrically conductive bacterial nanowires for promoting the EET process under anaerobic conditions, and the $c$-type cytochromes that are contained in the surface of the outer membrane are known to play an important role in the EET process $[5,6]$. 
With the advances in high-throughput technologies, a large number of studies have been constructed to investigate the EET process in S. oneidensis MR-1 from genome-wide expression profiles. Generally, these studies used the differential expression information from RNA-level gene expression datasets that were derived from different EET conditions [7-9]. For example, by using RNA sequencing (RNA-Seq) data, Barchinger et al. analyzed the differentially expressed genes in S. oneidensis MR-1 under limiting $\mathrm{O}_{2}$ conditions, and thereby identified the important genes that promoted the EET process during $\mathrm{O}_{2}$ limitation [9]. As it encompasses all the RNA transcribed within the cells, such transcriptome studies can powerfully represent regulatory changes in response to the switched EET process at the transcript level.

On the other hand, there are multilevel complex mechanisms involved in regulating the process of messenger RNA (mRNA) to protein, including post-transcriptional regulation, translational control and post-translational modifications (such as methylation, acetylation, phosphorylation, etc.) [10]. For these reasons, cells' protein and mRNA levels are not well correlated, as indicated by previous systemwide quantitative analyses of protein and mRNA expression [11,12]. Therefore, mRNA expression data has been unable to unambiguously relate biological processes to particular proteins alone. Meanwhile, the proteomics measurements have been shown to be more sensitively closed to the cells' states themselves, and have thereby served as an important complement to the transcriptome data for the analysis of changes in biological processes $[13,14]$. In addition, as the machines of life, proteins rarely work in isolation but rather interact with each other to form protein-protein interaction (PPI) networks to carry out biological processes [15-17]. As such, the construction of PPI networks to study protein functions of a specific biological process will be very effective.

Therefore, in the present paper, we used proteomics data and the relevant network-based methods to identify the key active proteins involved in the EET process in S. oneidensis MR-1. We firstly identified the active proteins involved in activating the EET process by clustering analysis of the proteomics data (Section 3.1). Then, we constructed active protein networks and identified the most important active proteins by network centralization analysis (Section 3.2). We further analyzed the active network motifs that are potentially involved in activating the EET process and studied the relevant proteins; we also discuss the functional modules that formed from these proteins (Section 3.3).

\section{Materials and Methods}

\subsection{Identification of Active Proteins}

Taylor et al. collected six groups of samples of S. oneidensis MR-1 under different $\mathrm{O}_{2}$ conditions (three for aerobic and three for anaerobic), and measured the protein expression levels for 4436 protein-coding genes by mass spectrometry [18]. We excluded the proteins that were not expressed (protein copies $=0$ ) across all of the six samples, and clustered the remaining proteins using the Bioconductor package Mfuzz; the cluster number (4) and the fuzzifier (1.5) were used [19]. In order to identify proteins that play an important role in the EET process, we focused on the proteins which sharply changed before and after the activation of the EET process (see Section 3.1).

\subsection{Protein-Protein Interaction}

The protein interaction information was obtained from the STRING (Search Tool for Recurring Instances of Neighbouring Genes) database [20,21]. The interactions were assigned confidence scores according to the quantity of evidence that supported them and, according to the recommendations of STRING, $0.4,07$, and 0.9 are the medium, high, and highest confidence score, respectively. To evaluate the effect of different confidence scores as the filtered thresholds, we used 0.4, 0.5, 0.6, 0.7, 0.8, and 0.9 as the total STRING protein interaction confidence scores for PPI filtering. The resultant PPI networks were used as the background networks, and the active protein networks were extracted from these background networks for the active proteins. KEGG (Kyoto Encyclopedia of Genes and 
Genomes) enrichment analysis and protein domain enrichment analysis was carried out using STRING online tools.

\subsection{Transcriptional Regulatory Interaction}

The full transcriptional regulatory information was obtained from the RegPrecise database [22], which contains 826 pair transcriptional regulatory interactions among 62 transcription factors and 678 target genes in S. oneidensis MR-1. The active regulatory interactions for the protein-coding genes in each active protein network were conducted; then, by using nodes to represent proteins (and the corresponding genes) and arcs to represent the interactions, we integrated the two kinds of networks.

\subsection{Network Centralization}

Centralization is a concept that is used to identify the relative importance of nodes in a given network, and there are many measures to describe the importance of nodes in a network. We engaged two widely used methods for network centralization analysis: degree centralization was often used to identify the hubs of the network, while betweenness centralization was usually used to identify the bottlenecks of the network. The average rank of the degree centralization and betweenness centralization was used to rank the relative importance of proteins, by using the R package igraph [23].

\subsection{Network Motifs and Functional Modules}

Since the interacted proteins should have a higher chance of possessing similar functions than the un-interacted proteins, both of the biological motifs and modules play an important role in understanding complex biological processes. The identification of motifs was carried out with the FANMOD tool [24], with the conventional parameters ( $p$ value $<0.05$, z score $>2$, etc.). The functional modules were extracted using the NetCarto program, which maximized the network modularity $\mathrm{M}$ by a simulated annealing method [25].

\section{Results and Discussion}

\subsection{Identification of Active Proteins Involved in Activating the EET Process}

Since EET inactivates under high $\mathrm{O}_{2}$ concentrations and activates when $\mathrm{O}_{2}$ levels were lowered, Taylor et al. generated six groups of sequential samples under altered $\mathrm{O}_{2}$ levels for proteomics data in S. oneidensis MR-1 (group 1-3 for aerobic conditions and group 4-6 for anaerobic conditions) [18]. To identify the specific active proteins involved in activating the EET process, we clustered the genome-wide proteomics data. As illustrated in Figure 1, four distinct clusters were obtained according to protein expression patterns with the Bioconductor package MFuzz [19]. Cluster 1 contained proteins with a sharp decrease in expression from sample 3 (S3) to sample 4 (S4); Cluster 2 contained proteins with a sharp increase in expression from S3 to S4; Cluster 3 contained proteins with an immediate decrease and then transform to a slower decrease in expression across the six samples; and Cluster 4 contained proteins with a long period of no change followed by a sharp increase in expression across the six samples (Figure 1). Since S3 was sampled from the last steady state under high- $\mathrm{O}_{2}$ conditions while $\mathrm{S} 4$ was sampled from the first steady state under low $-\mathrm{O}_{2}$ conditions, the severe changes between $\mathrm{S} 3$ and $\mathrm{S} 4$ should reflect the transition from high- $\mathrm{O}_{2}$ (i.e., inactivated EET process) to low $-\mathrm{O}_{2}$ (i.e., activated EET process). Therefore, we mainly focused on the proteins in the clusters with severe changes between S3 and S4. In other words, the proteins in Cluster 1 (1012) and Cluster 2 (811) could reflect the changes from an inactivated EET process to an activated EET process, and these 1823 proteins were identified as the active proteins involved in activating the EET process and which would help us better understand the EET process.

We then performed KEGG pathway enrichment analysis for these two clusters of proteins. There were 17 pathways enriched in the proteins in Cluster 1 (down-regulated expression), and 16 pathways enriched in the proteins in Cluster 2 (up-regulated expression). It should be noted 
that enrichment analysis was mainly used to retrieve the functional profile of a given gene/protein set (i.e., differentially expressed proteins here), which is generally performed by using statistical approaches to find classes of genes/proteins that are significantly over-represented (i.e., by comparison of their frequency to the whole genome) [26]. Therefore, the term "pathway" (or "metabolism") merely represents that many proteins that are part of a specific metabolic pathway are identified here; it is neither necessarily a complete pathway nor a particular part of it (see Supplementary Tables S1 and S2 for the detailed lists). As illustrated in Figure 2, the most common pathways, including metabolic pathways, biosynthesis of secondary metabolites, microbial metabolism in diverse environments, and carbon metabolism, presented the large changes (i.e., much more proteins enriched in these pathways). Furthermore, the other enriched pathways only presented in one cluster (down-regulated expression or up-regulated expression). Down-regulated pathways included biosynthesis of unsaturated fatty acids (6 enriched proteins, false discovery rate (FDR): $3.76 \times 10^{-2}$ ); butanoate metabolism (13 enriched proteins, FDR: $\left.2.50 \times 10^{-3}\right)$; citrate cycle (TCA cycle) $(16$ enriched proteins, FDR: $\left.9.39 \times 10^{-7}\right)$; fatty acid degradation $\left(9\right.$ enriched proteins, FDR: $\left.2.35 \times 10^{-3}\right)$; fatty acid metabolism (12 enriched proteins, FDR: $\left.3.11 \times 10^{-2}\right)$; geraniol degradation $(7$ enriched proteins, FDR: $\left.2.36 \times 10^{-3}\right)$; glutathione metabolism (12 enriched proteins, FDR: $\left.2.37 \times 10^{-2}\right)$; glycolysis/gluconeogenesis (16 enriched proteins, FDR: $\left.5.05 \times 10^{-4}\right)$; lysine degradation (5 enriched proteins, FDR: $\left.4.81 \times 10^{-2}\right)$; propanoate metabolism $\left(10\right.$ enriched proteins, FDR: $\left.6.75 \times 10^{-3}\right)$; pyruvate metabolism (14 enriched proteins, FDR: $\left.3.76 \times 10^{-2}\right)$; synthesis and degradation of ketone bodies ( 4 enriched proteins, FDR: $2.37 \times 10^{-2}$ ); and valine, leucine, and isoleucine degradation $\left(18\right.$ enriched proteins, FDR: $\left.5.47 \times 10^{-7}\right)$. Up-regulated pathways included: 2-oxocarboxylic acid metabolism (16 enriched proteins, FDR: $1.54 \times 10^{-5}$ ); alanine, aspartate, and glutamate metabolism $\left(16\right.$ enriched proteins, FDR: $\left.4.60 \times 10^{-5}\right)$; arginine and proline metabolism (13 enriched proteins, FDR: 0.0124); biosynthesis of amino acids (52 enriched proteins, FDR: $1.11 \times 10^{-11}$ ); glycine, serine, and threonine metabolism (18 enriched proteins, FDR: $\left.1.75 \times 10^{-6}\right)$; glyoxylate and dicarboxylate metabolism (19 enriched proteins, FDR: $\left.7.40 \times 10^{-6}\right)$; lysine biosynthesis $(7$ enriched proteins, FDR: $\left.4.31 \times 10^{-2}\right)$; methane metabolism $\left(17\right.$ enriched proteins, FDR: $\left.3.02 \times 10^{-5}\right)$; nitrogen metabolism $\left(6\right.$ enriched proteins, FDR: $\left.4.34 \times 10^{-2}\right)$; pantothenate and CoA biosynthesis $(8$ enriched proteins, FDR: $\left.4.07 \times 10^{-2}\right)$; porphyrin metabolism (15 enriched proteins, FDR: $\left.5.68 \times 10^{-4}\right)$; and valine, leucine, and isoleucine biosynthesis (10 enriched proteins, FDR: $4.29 \times 10^{-4}$ ).
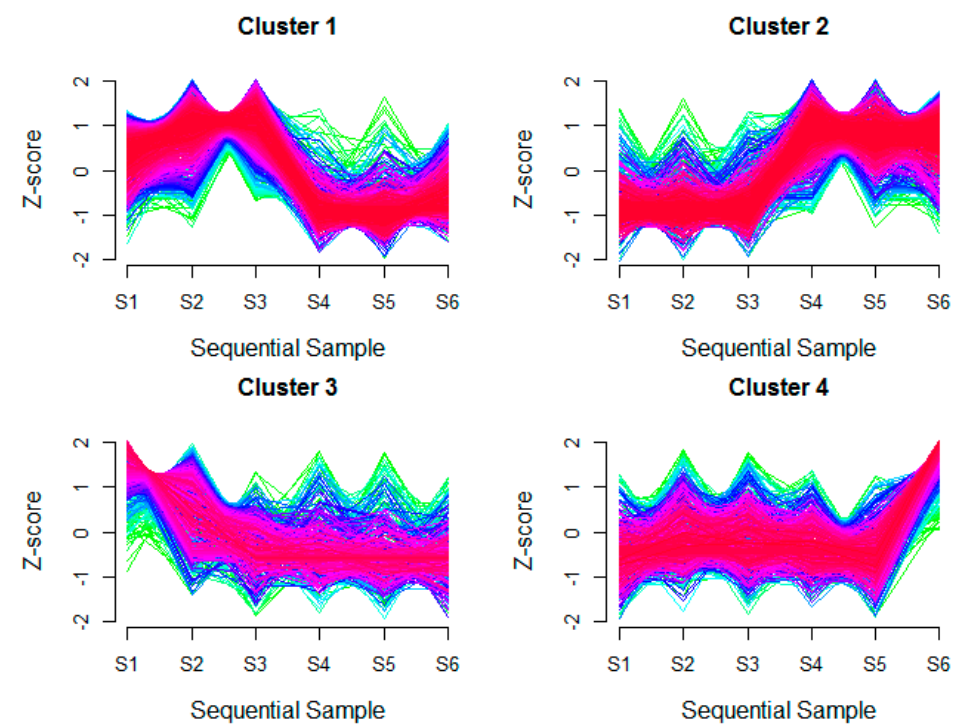

Figure 1. The protein expression patterns identified by MFuzz. Membership values are color-encoded from red (high values) to green (low values), S1-S6 represent six groups of sequential samples under different $\mathrm{O}_{2}$ levels (S1-S3 for high- $\mathrm{O}_{2}$ conditions, and $\mathrm{S} 4-\mathrm{S} 6$ for low- $\mathrm{O}_{2}$ conditions). 


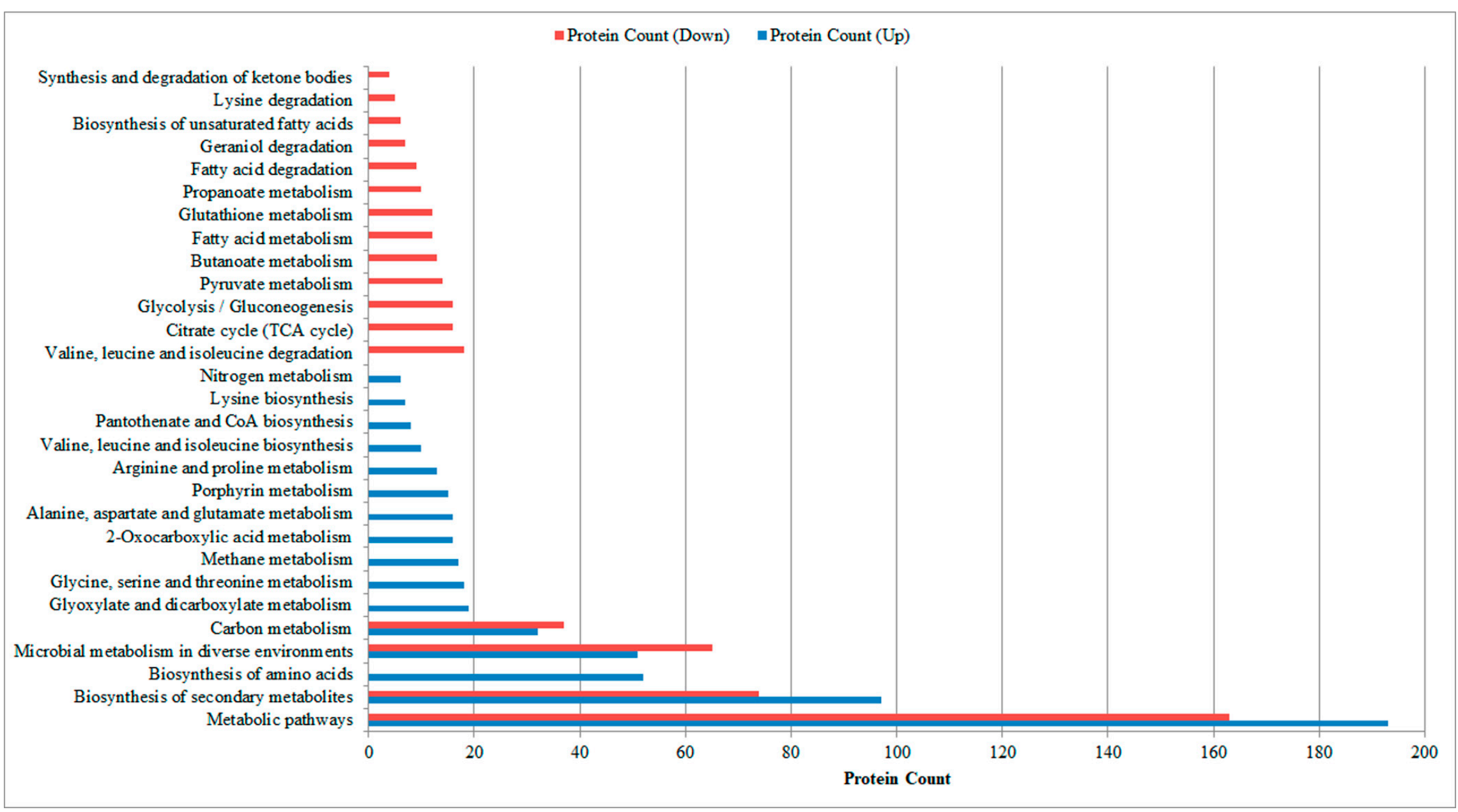

Figure 2. KEGG (Kyoto Encyclopedia of Genes and Genomes) pathway enrichment for the active proteins involved in activating the extracellular electron transfer (EET) process. False discovery rate $<0.05$. 
First of all, these results indicate that the metabolism is largely acclimated to the changed environmental condition $\left(\mathrm{O}_{2}\right.$ levels here), which is in agreement with the previous studies which used mRNA-level gene expression data for aerobic and anaerobic growth [9,27]. Secondly, except for the altered metabolic pathways, the biosynthesis of multiple amino acids was enriched in the up-regulated pathways; meanwhile, the degradation ones were enriched in the down-regulated pathways, in agreement with the expectation that $S$. oneidensis MR-1 needs to produce new proteins that acclimate to the new environment, which need different kinds of amino acids. Thirdly, the enriched, up-regulated pathway "porphyrin metabolism" and the involved active proteins (CobQ, Ftn, GltX, HemA, HemC, HemE, HemH, HemL, HemN, HemX, SO_0025, SO_0027, SO_2587, SO_3720, and SO_4208) intensively indicate that the heme processing, and, thereby, the c-type cytochrome biosynthesis, are needed in the limiting $\mathrm{O}_{2}$ condition. It should be noted that some of the protein-coding genes identified here were also identified as up-regulated ones in the previous transcriptome study [9], including HemA, HemH, HemL, HemN, SO_0027, SO_2587, SO_3720 and SO_4208. Furthermore, it is also worth noting that while the transcriptome study identified SO_4314 (HemD) [9], we identified the adjacent SO_4313 (HemC) and SO_4315 (HemX) here; such a simple yet important case will be helpful to demonstrating the importance and necessity of using proteome study to complement the transcriptome study.

\subsection{Networking of Active Protein Involved in Activating the EET Process}

Microbes can produce different proteins to respond to changing environmental conditions, and these proteins need to interact with each other to carry out specific biological processes. Such interacting protein systems can be represented and analyzed in the form of PPI networks, where nodes represent the proteins and edges represent interactions among the proteins $[15,16]$. Generally, networks describing a certain biological process (e.g., EET process here) depend on the biological contexts that underlie the biological process of interest [28]. Therefore, we need to construct subnetworks for these active proteins.

To achieve this, we firstly obtained genome-scale PPI information from STRING [20,21], and filtered the interactions with the total confidence scores. Enhancement of the filtered scores will result in greater loss of the PPIs, which will significantly affect the resulting PPI networks. As marked by STRING database, 0.4 is a medium confidence score, 0.7 is a high confidence score and 0.9 is the highest confidence score, and to test the robustness, we considered multiple confidence scores as the filtered thresholds for the comparison, from 0.4 to 0.9 , in increments of 0.1 . The resulting PPI networks were considered as the background networks, and we then educed the active subnetworks for the 1823 active proteins from these background networks. The final resultant active protein network information is summarized in Table 1.

Table 1. Statistics of the integrated networks used in this study.

\begin{tabular}{ccccccc}
\hline \multirow{2}{*}{ Confidence Score } & \multicolumn{2}{c}{ Protein Interaction } & Regulatory Interaction & \multicolumn{2}{c}{ Total } \\
\cline { 2 - 6 } & Proteins & Interactions & Genes & Interactions & Nodes & Arcs \\
\hline CS_0.4 & $1636\left(1711^{*}\right)$ & 17,577 & 582 & 714 & 1873 & 35,868 \\
CS_0.5 & $1577\left(1679^{*}\right)$ & 12,343 & 579 & 712 & 1815 & 25,398 \\
CS_0.6 & $1483\left(1613^{*}\right)$ & 9460 & 574 & 705 & 1728 & 19,625 \\
CS_0.7 & $1366\left(1520^{*}\right)$ & 7030 & 567 & 697 & 1617 & 14,757 \\
CS_0.8 & $1162\left(1310^{*}\right)$ & 4972 & 536 & 662 & 1408 & 10,606 \\
CS_0.9 & $997\left(1118^{*}\right)$ & 3112 & 502 & 618 & 1229 & 6842 \\
\hline
\end{tabular}

The node refers both to the gene and to the gene-encoded protein; the arc refers both to the regulatory interaction (a single direct arc) and to the protein interaction (two bidirectional arcs, or edges). ${ }^{*}$ indicates the numbers of the nodes that include the isolated ones.

To identify the most important active proteins in the EET process in S. oneidensis MR-1, we performed centralization analysis for these active protein networks. We firstly identified the 
top $2 \%$ key proteins in each active protein network (Table 2). Then, we used the frequencies of these key proteins to rank the top 20 key active proteins (Table 3). These 20 proteins were therefore considered to be the most important active proteins in the EET process; their biological functions were discussed below.

Table 2. The key active proteins in each active protein network.

\begin{tabular}{ccccccc}
\hline Rank & CS_0.4 & CS_0.5 & CS_0.6 & CS_0.7 & CS_0.8 & CS_0.9 \\
\hline 1 & SO_1325 & SO_1325 & SO_1325 & SO_1325 & SO_1325 & SO_0228 \\
2 & SO_3292 & SO_3292 & SO_3292 & SO_3292 & SO_0226 & SO_1325 \\
3 & SO_0435 & SO_3430 & SO_1126 & SO_3471 & SO_1207 & SO_2491 \\
4 & SO_3430 & SO_3440 & SO_3430 & SO_3430 & SO_2491 & SO_0226 \\
5 & SO_3440 & SO_1126 & SO_3471 & SO_3209 & SO_3471 & SO_0009 \\
6 & SO_1126 & SO_0435 & SO_0435 & SO_1126 & SO_3292 & SO_1207 \\
7 & SO_3471 & SO_3471 & SO_3440 & SO_0435 & SO_1926 & SO_2912 \\
8 & SO_2619 & SO_2619 & SO_2619 & SO_2491 & SO_3430 & SO_3292 \\
9 & SO_3432 & SO_3432 & SO_1207 & SO_1926 & SO_2406 & SO_0610 \\
10 & SO_4749 & SO_4749 & SO_3209 & SO_3440 & SO_1126 & SO_1677 \\
11 & SO_1197 & SO_1926 & SO_1926 & SO_4747 & SO_0236 & SO_3471 \\
12 & SO_0603 & SO_4215 & SO_4747 & SO_0226 & SO_0009 & SO_0237 \\
13 & SO_2411 & SO_4747 & SO_3432 & SO_4215 & SO_0610 & SO_3207 \\
14 & SO_1926 & SO_0603 & SO_4749 & SO_4749 & SO_3209 & SO_4428 \\
15 & SO_4215 & SO_0226 & SO_3441 & SO_1207 & SO_0435 & SO_3209 \\
16 & SO_3441 & SO_3209 & SO_4586 & SO_3432 & SO_2780 & SO_2619 \\
17 & SO_1207 & SO_0009 & SO_0770 & SO_2619 & SO_0237 & SO_1629 \\
18 & SO_4586 & SO_1197 & SO_0009 & SO_4586 & SO_4747 & SO_3210 \\
19 & SO_4747 & SO_1207 & SO_4215 & SO_0009 & SO_0608 & SO_0247 \\
20 & SO_3639 & SO_3441 & SO_0226 & SO_0228 & SO_0425 & SO_3639 \\
21 & SO_0226 & SO_4016 & SO_0610 & SO_2406 & SO_2619 & SO_3430 \\
22 & SO_1552 & SO_4586 & SO_1197 & SO_0610 & SO_1473 & SO_0435 \\
\hline
\end{tabular}

For the purpose of consistency, we considered $2 \%$ of 1118 (which is the minimum node number in the active protein networks) as the key proteins ( 22 proteins). Multiple confidence scores (CS) are used for the comparison, from 0.4 to 0.9 , in increments of 0.1 , as indicated by CS_0.x in the table.

Table 3. The top 20 key active proteins ranked by the frequencies of the key proteins in Table 2.

\begin{tabular}{ccccc}
\hline Rank & ID & Name & Number & Biological Function \\
\hline 1 & SO_0226 & RpsL & 6 & 30S ribosomal protein S12 \\
2 & SO_0435 & HemE & 6 & Uroporphyrinogen decarboxylase \\
3 & SO_1207 & RpsO & 6 & 30S ribosomal protein S15 \\
4 & SO_1325 & GltB & 6 & NADPH-dependent glutamate synthase large subunit GltB \\
5 & SO_2619 & MetG & 6 & Methionine-tRNA ligase \\
6 & SO_3292 & GuaA & 6 & GMP synthase [glutamine-hydrolyzing] \\
7 & SO_3430 & RecA & 6 & Protein RecA \\
8 & SO_3471 & GlyA & 6 & Serine hydroxymethyltransferase \\
9 & SO_0009 & DnaN & 5 & DNA polymerase III subunit beta \\
10 & SO_1126 & DnaK & 5 & Chaperone protein DnaK \\
11 & SO_1926 & GltA & 5 & Citrate synthase \\
12 & SO_3209 & CheY & 5 & Chemotaxis signal transduction system response regulator CheY \\
13 & SO_4747 & AtpD & 5 & ATP synthase subunit beta \\
14 & SO_0610 & PetC & 4 & Ubiquinol-cytochrome c reductase cytochrome c1 subunit PetC \\
15 & SO_3432 & RpoS & 4 & RNA polymerase sigma factor RpoS \\
16 & SO_3440 & Eno & 4 & Enolase \\
17 & SO_4215 & FtsZ & 4 & Cell division protein FtsZ \\
18 & SO_4586 & FtsY & 4 & Signal recognition particle receptor FtsY \\
19 & SO_4749 & AtpA & 4 & ATP synthase subunit alpha \\
20 & SO_1197 & FtsH & 3 & ATP-dependent zinc metalloprotease FtsH \\
\hline
\end{tabular}

NADPH: nicotinamide adenine dinucleotide phosphate; tRNA: transfer RNA; GMP: guanosine monophosphate; ATP: adenosine triphosphate. 
First of all, metabolism- and energy-related proteins are the most abundant ones in these 20 proteins $(7$ in 20$)$. On the basis of the annotated information in the universal protein resource database (UniProt) [29], GuaA (Rank 6) catalyzes the synthesis of guanosine monophosphate (GMP), which is involved in purine metabolism; GlyA (Rank 8) catalyzes the reversible conversion of serine and glycine, which serves as the major source of one-carbon groups required for the biosynthesis of purines, thymidylate, methionine, and other important biomolecules; GltA (Rank 11) is involved in the synthesis of isocitrate from oxaloacetate, which is known to be a part of the tricarboxylic acid cycle pathway; Eno (Rank 16) catalyzes the reversible conversion of 2-phosphoglycerate into phosphoenolpyruvate, and is essential for the degradation of carbohydrates via glycolysis. AtpA and AtpD (Rank 13 and 19) are two subunits of adenosine triphosphate (ATP) synthase; the alpha subunit AtpA primarily plays a regulatory role, while the beta subunit AtpD hosts the catalytic sites. They are involved in producing ATP in the presence of a proton gradient across the cellular membrane; protein RecA (Rank 7) can catalyze the hydrolysis of ATP.

Secondly, the signal and transcriptional regulatory proteins are also very abundant (6 in 20). It is presumably because of this that the transcriptional regulation of genes will respond to the changed environment conditions around cells, such as change in the concentration and activity of intracellular molecules, and transform extracellular signals into specific intracellular molecular activity. GltB (Rank 4) is known to be the nicotinamide adenine dinucleotide phosphate (NADPH)-dependent glutamate synthase; protein expression data showed that it was greatly enhanced in expression after the EET pathway was activated, which seems to have a direct connection with the fact that glutamate can be used as the signal transmitter [30]. Our recent studies have also shown that GltB works at the center of the signal processing unit, which can help transmit signals to the EET-related transcription factors and cofactors as well as EET target proteins [31]. CheY (Rank 12) is a signal transduction system response regulator, while FtsY (Rank 18) is a signal recognition particle receptor. DNA polymerase III DnaN (Rank 9) is required for initiation and processivity of DNA replication; DnaK (Rank 10) acts as a chaperone protein which provides stability in the transcriptional regulation process [32]; and RpoS (Rank 15) is an RNA polymerase sigma factor which can be used to coordinate transcription factors through protein-protein interactions [33].

Thirdly, from the rank order viewpoint (see Table 3), several translation-related proteins are also very important; this further agrees that $S$. oneidensis MR-1 needed to produce new proteins to acclimate to the new environment, which also suggests that although many microbial proteins are regulated at the transcription level, the regulation of the translation level is also a vital mechanism. RpsL (Rank 1) and RpsO (Rank 3) are directly responsible for translation, as they play an important role in translational accuracy. Speaking specifically, RpsL interacts with and stabilizes bases of the $16 \mathrm{~S}$ ribosomal RNA (rRNA) that are involved in transfer RNA (tRNA) selection at the A site and with the mRNA backbone [34], and RpsO is one of the primary rRNA binding proteins, binding directly to $16 \mathrm{~S}$ rRNA where it helps nucleate assembly of the platform of the $30 \mathrm{~S}$ subunit by binding and bridging several RNA helices of the 16S rRNA [35]. Furthermore, MetG (Rank 5) is required for both of the initiation of all mRNA translation and the elongation of protein synthesis.

Lastly, as we expected, several key active proteins are also directly linked to the EET process. Uroporphyrinogen decarboxylase HemE (Rank 2) is known to be involved in protoporphyrin-IX biosynthesis, and is therefore an important biosynthesis protein for $c$-type cytochromes, which are the main functional molecules involved in the EET process [8]. PetC (Rank 14) is another important protein involved in the EET process, it has been found playing critical roles in both aerobic and anaerobic respiration with highly toxic metals as electron acceptor [36]. This protein also presented various kinds of molecular function, such as electron carrier activity, electron transport, heme binding and metal ion binding, etc. ATP-dependent zinc metalloprotease FtsH (Rank 20) should also be involved in the EET process, considering that targeting metalloproteases has been used in redox modulation [37]. 


\subsection{Active Network Motifs Involved in Activating the EET Process}

To understand the mechanisms of gene regulation for the protein-coding genes involved in the EET process, the transcriptional regulatory interactions associated with active proteins were examined. We firstly obtained the transcriptional regulatory information for S. oneidensis MR- 1 from the RegPrecise database [22]. Then, we educed the active regulatory interactions for each active protein network obtained in Section 3.2 (see also Table 1). Lastly, by using nodes to represent proteins (and the corresponding genes) and arcs to represent the interactions, we constructed the integrated protein interaction and transcriptional regulatory networks for the 1823 active proteins (Table 1).

Biological networks, including regulatory networks, protein networks, and the integrated ones, often contain many small but over-represented motifs that form essential functional units of biological processes in cells [38]. At first glance, there must be active network motifs involved in activating the EET process, and the proteins involved in such active motifs should be as important as those identified by network centralization analysis. The network motif analysis tool FANMOD was then used to detect the three-node network motifs in these integrated networks. As illustrated in Table 4, 10 kinds of three-node network motifs were detected in these integrated networks. While three of them were presented in only one network (Table 4, Motif ID 7, 9, and 10), the other seven were presented in all (or most for motif 8) of these networks, and they were therefore considered as active network motifs.

Furthermore, to reveal the exclusive active network motifs involved in activating the EET process, we compared these seven active network motifs to the highly conserved ones in the Shewanella species, which were identified by comparative analysis of the integrated networks of 13 Shewanella species in our recent study [39]. We found that four of them were the highly conserved motifs in the Shewanella species. Their biological functions have been well discussed: (1) The motif Co-regulated PPI (Table 4, Motif ID 1) played an important role in the "standby mode" of protein utilization, which helps cells to rapidly respond to changing environmental conditions [39-42]. (2) The motif Protein Clique (Table 4, Motif ID 2) was expected to capture only some local, physically interacted components; such motifs could be used to build complex assemblies, which usually correspond to a multicomponent protein machine [43]. (3) The main function of the Co-regulated Proteins (Table 4, Motif ID 3) was to allow the coordinated expression of a group of genes with shared function [38]. (4) The motif PPI Regulating (Table 4, Motif ID 4) represented a transcription regulator that was made of a complex of two proteins, which meant that the transcription factor (TF) required another TF (or cofactor) for their activity [44].

On the other hand, the remaining three motifs (Table 4, Motif ID 5, 6, and 8) should be considered as the exclusive active network motifs involved in activating the EET process, and the proteins involved in these active motifs may reflect the important changes of the EET process (i.e., from inactivation to activation). Therefore, we identified the relevant proteins in these active motifs and discussed their potential roles in the EET process. To achieve this, we used the network constructed with STRING's confidence score 0.4 , as it contained the largest number of such active proteins. A total of 191 active proteins are involved in these three important motifs. We performed further domain enrichment analysis for these active proteins (Figure 3A). Two kinds of important results emerged from the domain enrichment analysis. Firstly, the multiheme cytochrome is enriched as it is a key component of the electron transfer channel in S. oneidensis MR-1. Previous studies have shown that the multiheme cytochromes can work together to cause the long distance redox chain that ranges from the cell inner membrane to the extracellular space. For example, the two cytochromes MtrA and MtrC can be stabilized by an outer membrane porin MtrB to form the stable MtrCAB complex, the main function of which is to allow electrons to transfer from the inner membrane CymA to the extracellular OmcA through the MtrCAB complex [45-50]. Secondly, a large number of signal domains are enriched, which is consistent with recent study that the signal proteins may contribute to the coordination of EET-related transcription factors to trigger a large number of conditional responses in the EET process [31], and a multicomponent signaling network involved in the transformation from aerobic conditions to anaerobic conditions has also been reported [51]. In addition, the comparative analysis 
of the proportion of the EET proteins and signal proteins in these active proteins to the whole genome of $S$. oneidensis MR-1 also resulted in the same conclusions (Figure 3B).

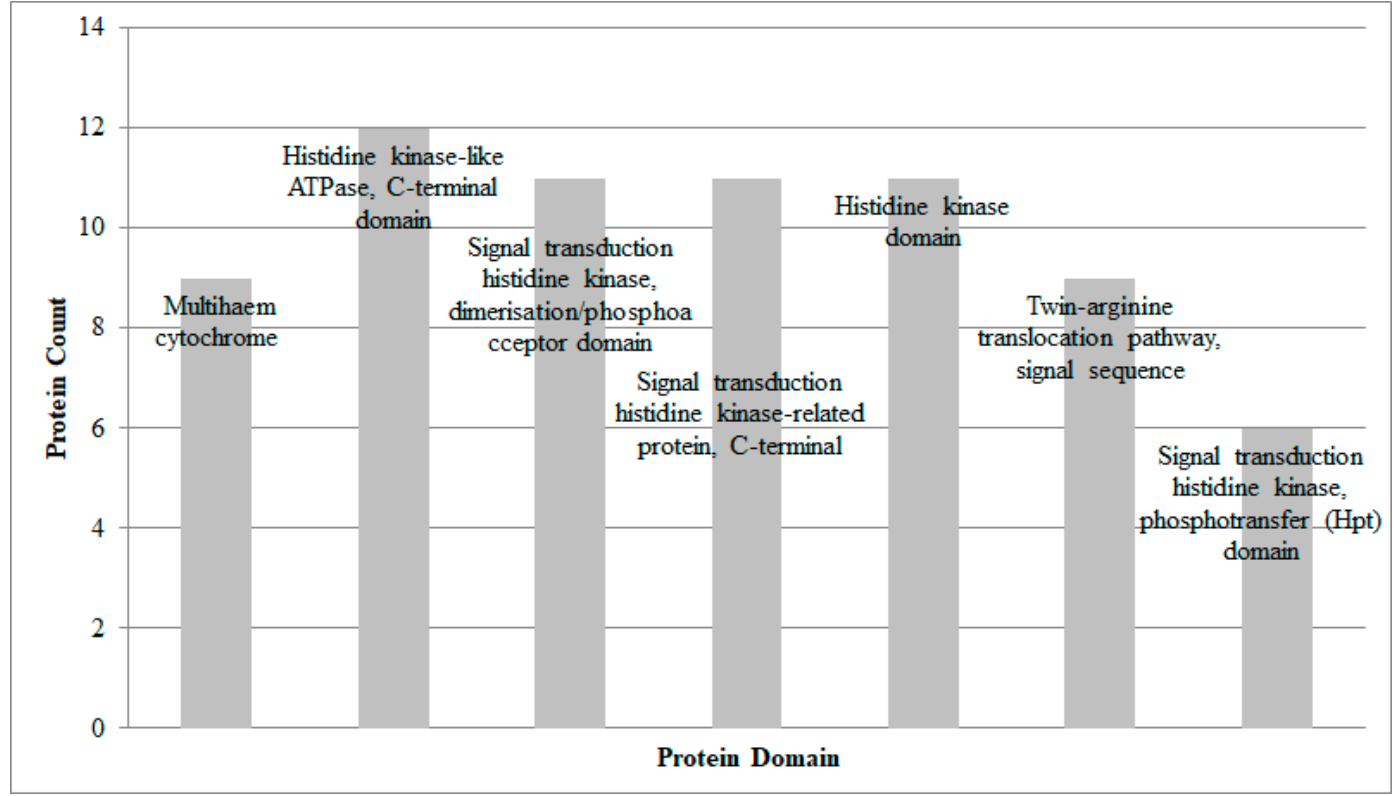

(A)

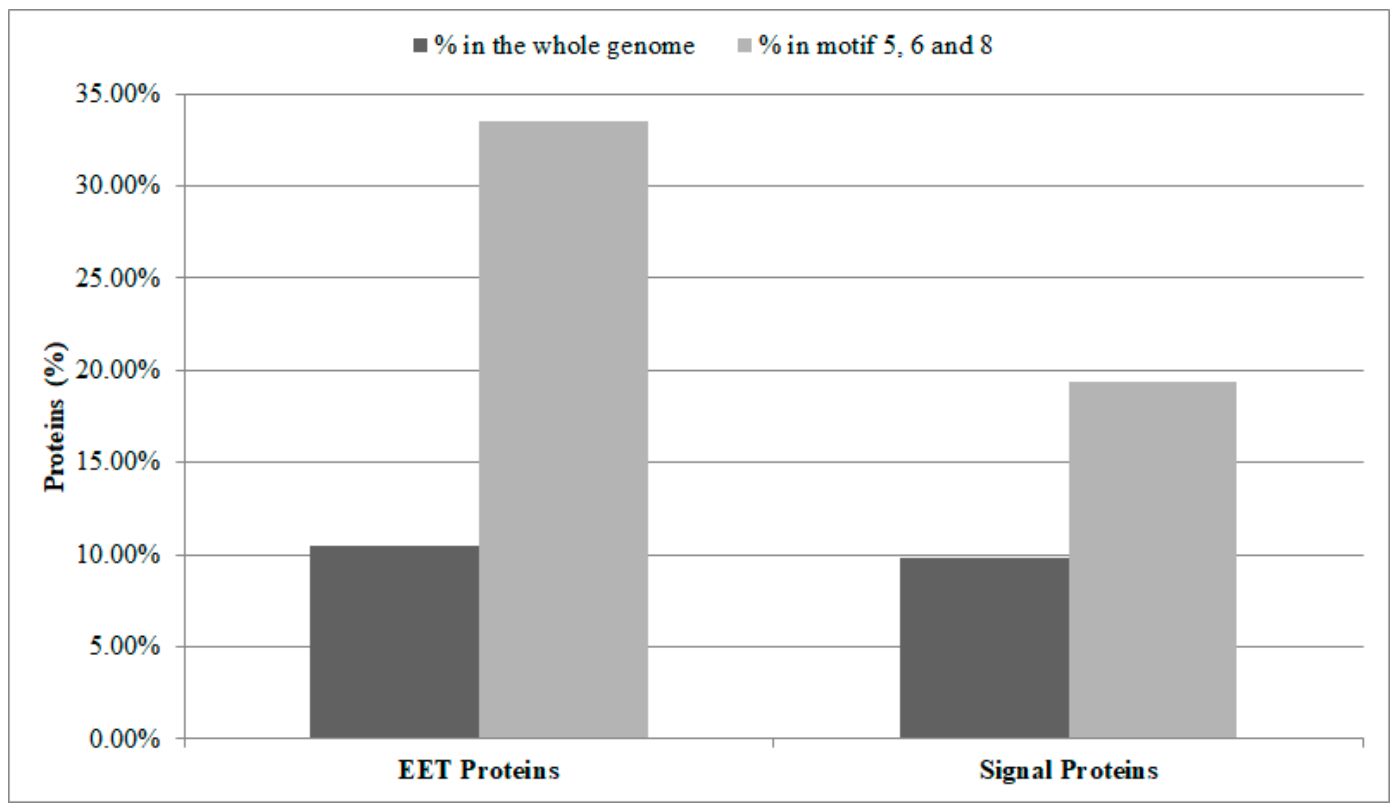

(B)

Figure 3. The proteins involved in network motifs 5, 6, and 8. (A) The protein domain enrichment, false discovery rate $<0.05$. (B) The EET proteins and signal proteins (\%) in network motifs 5,6 , and 8 vs. those in the whole genome of Shewanella oneidensis MR-1. 
Table 4. Three-node active network motifs that are identified in the active integrated networks.

\begin{tabular}{|c|c|c|c|c|c|c|c|c|c|}
\hline ID & Motif Name & Illustration & Times & $\begin{array}{l}\text { Z-Score } \\
\text { (CS_0.4) }\end{array}$ & $\begin{array}{l}\text { Z-Score } \\
\text { (CS_0.5) }\end{array}$ & $\begin{array}{l}\text { Z-Score } \\
\text { (CS_0.6) }\end{array}$ & $\begin{array}{l}\text { Z-Score } \\
\text { (CS_0.7) }\end{array}$ & $\begin{array}{l}\text { Z-Score } \\
\text { (CS_0.8) }\end{array}$ & $\begin{array}{l}\text { Z-Score } \\
\text { (CS_0.9) }\end{array}$ \\
\hline 1 & Co-regulated PPI & & 6 & 10,462 & 6589.7 & 11,844 & 5282.5 & 5888.5 & 2087.4 \\
\hline 2 & Protein Clique & & 6 & 191.25 & 240.78 & 230.85 & 370.21 & 389.55 & 643.88 \\
\hline 3 & Co-regulated Proteins & & 6 & 166.86 & 215.43 & 214.67 & 352.11 & 367.41 & 624.95 \\
\hline 4 & PPI Regulating & & 6 & 61.387 & 61.452 & 68.695 & 51.728 & 46.724 & 28.317 \\
\hline 5 & Bi-feedforward Loop & & 6 & 6.5625 & 6.4191 & 6.6984 & 7.0355 & 6.1486 & 4.7833 \\
\hline 6 & Regulatory Cascade with a Feedback & & 6 & 5.1718 & 5.4026 & 5.9498 & 7.1723 & 7.0436 & 10.383 \\
\hline 7 & Regulated PPI & & 1 & 4.0703 & - & - & - & - & - \\
\hline 8 & Feedback with a PPI & & 5 & 3.0341 & 2.4203 & 3.3099 & 4.9955 & 6.0888 & - \\
\hline 9 & Bi-regulated Protein & & 1 & - & - & - & - & - & 2.288 \\
\hline 10 & Regulatory Cascade & $\bullet \rightarrow$ & 1 & - & - & - & - & - & 2.2782 \\
\hline
\end{tabular}

PPI: protein-protein interaction. Multiple confidence scores (CS) are used for the comparison, from 0.4 to 0.9 , in increments of 0.1 , as indicated by CS $0 . x$ in the table. 
These active proteins clearly indicated that the remaining three network motifs (Table 4, Motif ID 5, 6, and 8) reflected the biological changes for the EET process from inactivation to activation, and, accordingly, these proteins should also be regarded as the potential targets for EET-related studies. Therefore, we also constructed a protein network for these active proteins (Figure 4A), and analyzed the functional modules in the largest connected part of this network (Figure 4B). To our surprise, the proteins with multiheme cytochrome or multiple signal domains were not just enriched in these active proteins, they actually formed two functional modules (Figure 4C,D). The emphasis was therefore mainly placed on these two modules.

As shown in Figure 4C, the well-known MtrCAB pathway proteins (CymA, MtrA, MtrB, MtrC, and OmcA) formed a small cluster, together with the outer membrane porin Omp35 (SO_3896) and a secretion protein GspD (SO_0166). The porin Omp35 is markedly up-regulated anaerobically, and it has been shown to affect anaerobic electron transfer in an indirect manner [52], while the type II secretion system component GspD has also been related to the EET process in the reduction of external Mn(IV) and Fe(III) oxides [53]. Although some $c$-type cytochromes (i.e., CytcB, FccA, MtrD, NrfA, and ScyA) as well as the related cytochrome maturation system proteins (i.e., $\mathrm{CcmA}$ and $\mathrm{CcmD}$ ) in this module were also identified by the previous transcriptome study [9], the other proteins in the module should also be properly considered according to our proteomic results, including the $c$-type cytochrome $\mathrm{CcoO}$, CcoP, PetC, and SO_3420, the related oxidase/reductase (e.g., CydA and PetA) or a cytochrome $b$ (PetB), as well as SspA. For example, the $c$-type cytochrome $\mathrm{CcoO}$ and PetC have been shown to play a critical role in both aerobic and anaerobic respiration with highly toxic metals as electron acceptors [36] (see also previous centralization analysis), while SspA (SO_0611) is a transcriptional activator which lies beside the petABC operon (SO_0608-SO_0610) according to the RegPrecise database and might thereby be involved in activating the transcription of this gene cluster.

For the multisignal processing module in Figure 4D, there were three hubs (Crp, NarP, and RstA) in the clusters. While Crp and NarP were well-known for their roles in the EET process, the third hub, two-component signal transduction system response regulator RstA (SO_3594), has not been reported to be involved in the EET process. From the annotated function view, such a regulator is involved in controlling the production of curli, which are a kind of proteinaceous extracellular fibers. It is now acknowledged that the nanowires are extensions of the outer membrane, rather than pilin-based structures [6]. Nevertheless, the formation of biofilm on the S. oneidensis MR-1 surface has also been shown to enhance the efficiency of electron transfer [54,55], and considering that curli are the major component of the extracellular matrix involved in bacterial biofilm formation [56], it is thereby tempting to speculate that RstA will be potentially implicated in the EET process. The remaining 34 proteins in this module are mostly signal processing proteins. Among them, only a few proteins have been identified by the previous transcriptome study (e.g., RpoE is considered to signal the initial response to $\mathrm{O}_{2}$ limitation) [9]; the RNA polymerase sigma factor RpoS can be used to coordinate transcription factors [33] (see also previous centralization analysis). The signal proteins SO_2145 and SO_1417 have been shown to play a central role in triggering the EET pathways under anaerobic environments $[31,57,58]$. The others are mostly not well understood, including response regulators (e.g., SO_0622, SO_2127), histidine kinases (e.g., SO_0352, SO_1327), sensory box proteins (e.g., SO_0341, SO_0569), etc. However, as response regulators and histidine kinases are generally involved in the basic stimulus-response processes that allow microorganisms to sense and respond to environmental changes [59], such an overabundance of signal proteins is therefore believed to be helpful in deciphering how Shewanella elicited a wide range of condition-specific responses under the changed environmental conditions. Furthermore, it is also reasonable to relate the sensory box proteins in this module to the EET process, considering that the deletion mutant of the Shewanella sensory box protein SO_3389 cannot grow with several extracellular electron acceptors [60].

Taken together, the studies on the interactions of motif-guided active proteins and the related functional modules also helped us to recapitulate known EET proteins as well as predict new ones. In addition to the $c$-type cytochromes and the related regulators (such as Crp and NarP), we have 
also identified a considerable number of signal processing proteins potentially implicated in the EET process. The resulting active proteins (especially those unreported ones) should be therefore regarded as potential targets in the future EET-related studies. In future steps, we will focus on how Shewanella senses and responds to environmental changes (with these signal proteins), and how Shewanella coordinates the transcriptional regulation and protein interaction involved in the EET process.

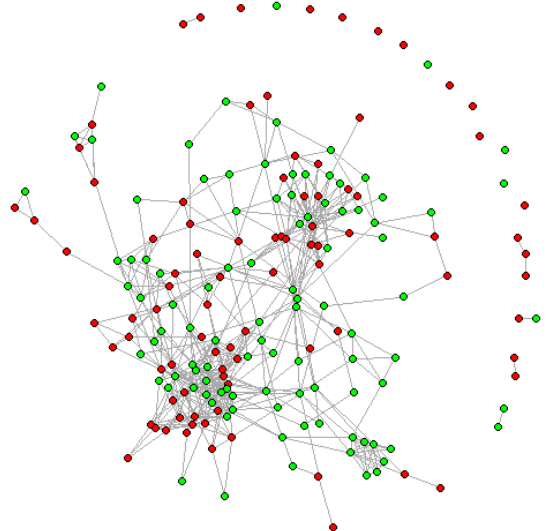

(A)

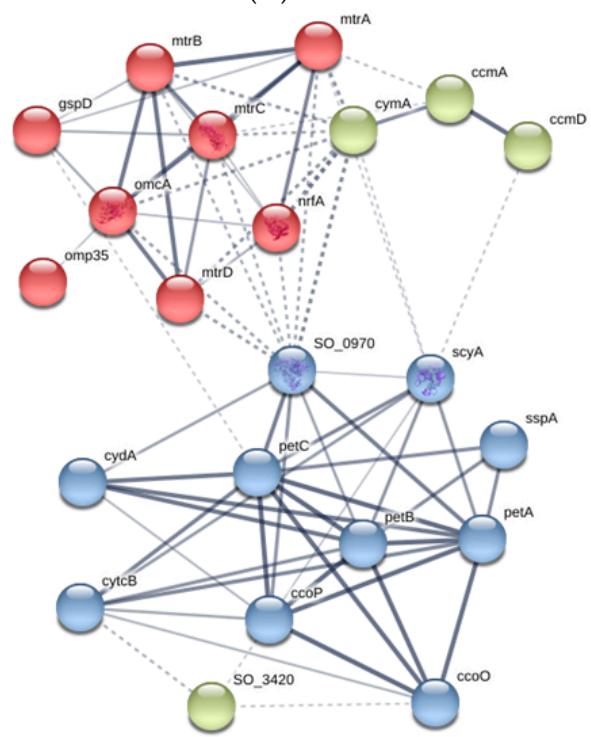

(C)

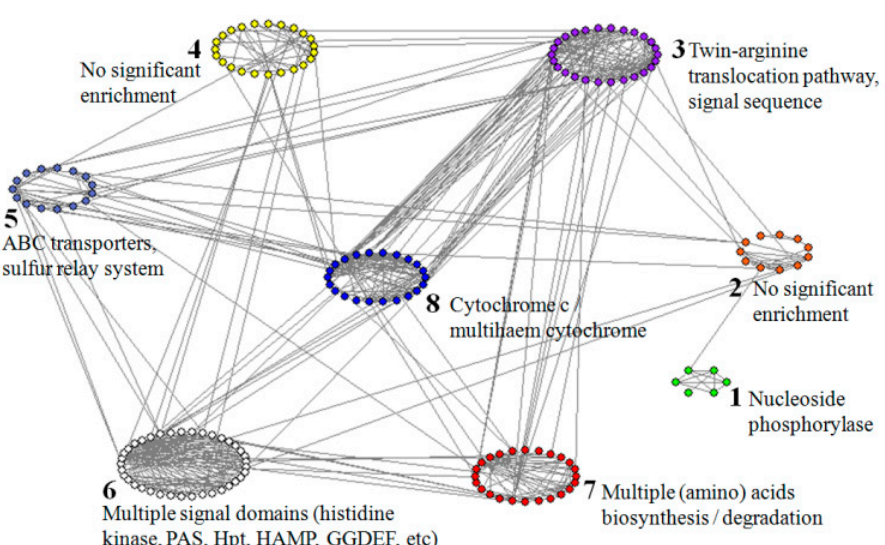

(B)

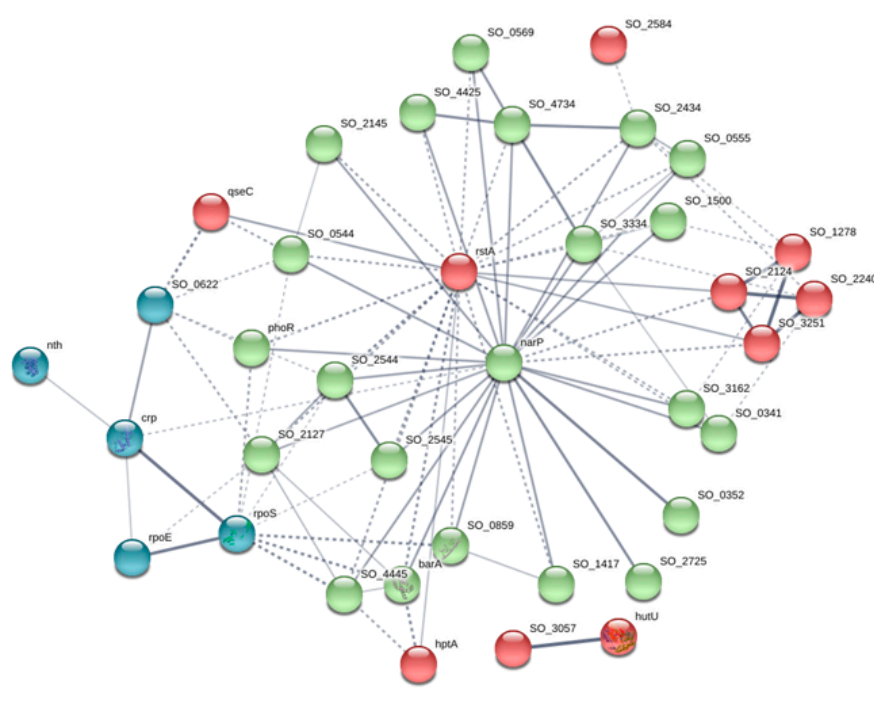

(D)

Figure 4. The functional modules in the proteins involved in network motifs 5, 6, and 8. (A) The protein interaction network generated by the R package igraph [23]; green nodes refer to the down-regulated proteins and red nodes refer to the up-regulated proteins. (B) The functional modules in the largest connected part of the protein interaction network; drawn by the Pajek program [61], modules are shown in distinct colors. (C) Module 8: multiheme cytochromes, and (D) Module 6: multisignal processing, which are generated by using the default parameters for the k-means cluster of these two modules in STRING (Search Tool for Recurring Instances of Neighbouring Genes) online tool [20,21], clusters are shown in distinct colors.

\section{Conclusions}

To improve the electron transfer efficiency of electricigens using genetic engineering technology, there is a need to understand and elucidate the molecular mechanism of the EET process, such as by discovering the key proteins involved in the EET process. A combinatorial approach utilizing proteomics data, clustering analysis, PPI networks, network centralization analysis, network motifs, and functional modules has been carried out to identify the key active proteins capable of activating 
the EET process in the present study. A total of 1823 proteins were found to be associated with the changed EET process by clustering analysis. These active proteins were then networked, and the top 20 key active proteins were identified by network centralization analysis; these proteins may serve as prospective targets for experimental confirmation. Furthermore, the active proteins involved in three exclusive EET-related active network motifs were identified, and their interactions as well as the accompanying functional modules were also discussed, which further support that the network-based methods could help to identify the key active proteins for future EET-related studies.

Supplementary Materials: The following are available online at www.mdpi.com/2073-4425/9/1/41/s1. Table S1: Complete list of the KEGG pathway enrichment analysis for the down-regulated proteins in Cluster 1, Table S2: Complete list of the KEGG pathway enrichment analysis for the up-regulated proteins in Cluster 2.

Acknowledgments: The work was supported by the National Natural Science Foundation of China (No. 61472078), the Key Research \& Development Program of Jiangsu Province (BE2016002-3), the Open Research Fund of State Key Laboratory of Bioelectronics from Southeast University, and the Natural Science Foundation of Anhui Education Department (KJ2015A264, KJ2015A290).

Author Contributions: D.D. designed the study, carried out the study, and drafted the manuscript; X.S. conceived of the study and wrote the manuscript. All authors read and approved the final manuscript.

Conflicts of Interest: The authors declare no conflict of interest.

\section{References}

1. Hau, H.H.; Gralnick, J.A. Ecology and biotechnology of the genus Shewanella. Annu. Rev. Microbiol. 2007, 61, 237-258. [CrossRef] [PubMed]

2. Shi, L.; Dong, H.; Reguera, G.; Beyenal, H.; Lu, A.; Liu, J.; Yu, H.Q.; Fredrickson, J.K. Extracellular electron transfer mechanisms between microorganisms and minerals. Nat. Rev. Microbiol. 2016, 14, 651-662. [CrossRef] [PubMed]

3. Wu, D.; Xing, D.; Lu, L.; Wei, M.; Liu, B.; Ren, N. Ferric iron enhances electricity generation by Shewanella oneidensis MR-1 in MFCs. Bioresour. Technol. 2013, 135, 630-634. [CrossRef] [PubMed]

4. Jiang, Y.; Liang, P.; Liu, P.; Wang, D.; Miao, B.; Huang, X. A novel microbial fuel cell sensor with biocathode sensing element. Biosens. Bioelectron. 2017, 94, 344-350. [CrossRef] [PubMed]

5. El-Naggar, M.Y.; Wanger, G.; Leung, K.M.; Yuzvinsky, T.D.; Southam, G.; Yang, J.; Lau, W.M.; Nealson, K.H.; Gorby, Y.A. Electrical transport along bacterial nanowires from Shewanella oneidensis MR-1. Proc. Natl. Acad. Sci. USA 2010, 107, 18127-18131. [CrossRef] [PubMed]

6. Pirbadian, S.; Barchinger, S.E.; Leung, K.M.; Byun, H.S.; Jangir, Y.; Bouhenni, R.A.; Reed, S.B.; Romine, M.F.; Saffarini, D.A.; Shi, L.; et al. Shewanella oneidensis MR-1 nanowires are outer membrane and periplasmic extensions of the extracellular electron transport components. Proc. Natl. Acad. Sci. USA 2014, 111, 12883-12888. [CrossRef] [PubMed]

7. Beliaev, A.S.; Klingeman, D.M.; Klappenbach, J.A.; Wu, L.; Romine, M.F.; Tiedje, J.M.; Nealson, K.H.; Fredrickson, J.K.; Zhou, J. Global transcriptome analysis of Shewanella oneidensis MR-1 exposed to different terminal electron acceptors. J. Bacteriol. 2005, 187, 7138-7145. [CrossRef] [PubMed]

8. Yang, Y.; Harris, D.P.; Luo, F.; Xiong, W.; Joachimiak, M.; Wu, L.; Dehal, P.; Jacobsen, J.; Yang, Z.; Palumbo, A.V. Snapshot of iron response in Shewanella oneidensis by gene network reconstruction. BMC Genom. 2009, 10, 131. [CrossRef] [PubMed]

9. Barchinger, S.E.; Pirbadian, S.; Sambles, C.; Baker, C.S.; Leung, K.M.; Burroughs, N.J.; El-Naggar, M.Y.; Golbeck, J.H. Regulation of Gene Expression in Shewanella oneidensis MR-1 during Electron Acceptor Limitation and Bacterial Nanowire Formation. Appl. Environ. Microbiol. 2016, 82, 5428-5443. [CrossRef] [PubMed]

10. Gingold, H.; Pilpel, Y. Determinants of translation efficiency and accuracy. Mol. Syst. Biol. 2011, 7, 481. [CrossRef] [PubMed]

11. Taniguchi, Y.; Choi, P.J.; Li, G.-W.; Chen, H.; Babu, M.; Hearn, J.; Emili, A.; Xie, X.S. Quantifying E. coli Proteome and Transcriptome with Single-Molecule Sensitivity in Single Cells. Science 2010, 329, 533-538. [CrossRef] [PubMed]

12. Zhao, Q.; Stettner, A.I.; Reznik, E.; Paschalidis, I.C.; Segre, D. Mapping the landscape of metabolic goals of a cell. Genome Biol. 2016, 17. [CrossRef] [PubMed] 
13. Baldrian, P.; Lopez-Mondejar, R. Microbial genomics, transcriptomics and proteomics: New discoveries in decomposition research using complementary methods. Appl. Microbiol. Biotechnol. 2014, 98, 1531-1537. [CrossRef] [PubMed]

14. Wang, J.; Islam, F.; Li, L.; Long, M.; Yang, C.; Jin, X.; Ali, B.; Mao, B.; Zhou, W. Complementary RNA-Sequencing Based Transcriptomics and iTRAQ Proteomics Reveal the Mechanism of the Alleviation of Quinclorac Stress by Salicylic Acid in Oryza sativa ssp. japonica. Int. J. Mol. Sci. 2017, 18, 1975. [CrossRef] [PubMed]

15. Barabasi, A.L.; Oltvai, Z.N. Network biology: Understanding the cell's functional organization. Nat. Rev. Genet. 2004, 5, 101-113. [CrossRef] [PubMed]

16. Vidal, M.; Cusick, M.E.; Barabasi, A.L. Interactome Networks and Human Disease. Cell 2011, 144, $986-998$. [CrossRef] [PubMed]

17. Chen, L.; Pan, H.; Zhang, Y.H.; Feng, K.; Kong, X.; Huang, T.; Cai, Y.D. Network-Based Method for Identifying Co-Regeneration Genes in Bone, Dentin, Nerve and Vessel Tissues. Genes 2017, 8, 252. [CrossRef] [PubMed]

18. Taylor, R.C.; Robertson, B.-J.M.W.; Markillie, L.M.; Serres, M.H.; Linggi, B.E.; Aldrich, J.T.; Hill, E.A.; Romine, M.F.; Lipton, M.S.; Wiley, H.S. Changes in translational efficiency is a dominant regulatory mechanism in the environmental response of bacteria. Integr. Biol. 2013, 5, 1393-1406. [CrossRef] [PubMed]

19. Kumar, L.; Futschik, M.E. Mfuzz: A software package for soft clustering of microarray data. Bioinformation 2007, 2, 5-7. [CrossRef] [PubMed]

20. Franceschini, A.; Szklarczyk, D.; Frankild, S.; Kuhn, M.; Simonovic, M.; Roth, A.; Lin, J.; Minguez, P.; Bork, P.; von Mering, C.; et al. STRING v9.1: Protein-protein interaction networks, with increased coverage and integration. Nucleic Acids Res. 2013, 41, D808-D815. [CrossRef] [PubMed]

21. Szklarczyk, D.; Franceschini, A.; Wyder, S.; Forslund, K.; Heller, D.; Huerta-Cepas, J.; Simonovic, M.; Roth, A.; Santos, A.; Tsafou, K.P.; et al. STRING v10: Protein-protein interaction networks, integrated over the tree of life. Nucleic Acids Res. 2015, 43, D447-D452. [CrossRef] [PubMed]

22. Novichkov, P.S.; Kazakov, A.E.; Ravcheev, D.A.; Leyn, S.A.; Kovaleva, G.Y.; Sutormin, R.A.; Kazanov, M.D.; Riehl, W.; Arkin, A.P.; Dubchak, I.; et al. RegPrecise 3.0-A resource for genome-scale exploration of transcriptional regulation in bacteria. BMC Genom. 2013, 14, 745. [CrossRef]

23. Csardi, G.; Nepusz, T. The igraph software package for complex network research. InterJournal 2006, CX.18, 1695.

24. Wernicke, S.; Rasche, F. FANMOD: A tool for fast network motif detection. Bioinformatics 2006, 22, 1152-1153. [CrossRef] [PubMed]

25. Guimera, R.; Nunes Amaral, L.A. Functional cartography of complex metabolic networks. Nature 2005, 433, 895-900. [CrossRef] [PubMed]

26. Hung, J.H. Gene Set/Pathway enrichment analysis. Methods Mol. Biol. 2013, 939, 201-213. [PubMed]

27. Pinchuk, G.E.; Hill, E.A.; Geydebrekht, O.V.; De Ingeniis, J.; Zhang, X.; Osterman, A.; Scott, J.H.; Reed, S.B.; Romine, M.F.; Konopka, A.E.; et al. Constraint-based model of Shewanella oneidensis MR-1 metabolism: A tool for data analysis and hypothesis generation. PLoS Comput. Biol. 2010, 6, e1000822. [CrossRef] [PubMed]

28. Ideker, T.; Krogan, N.J. Differential network biology. Mol. Syst. Biol. 2012, 8, 565. [CrossRef] [PubMed]

29. The UniProt Consortium. UniProt: The universal protein knowledgebase. Nucleic Acids Res. 2017, 45, D158-D169.

30. Saitoh, F.; Wakatsuki, S.; Tokunaga, S.; Fujieda, H.; Araki, T. Glutamate signals through mGluR2 to control Schwann cell differentiation and proliferation. Sci. Rep. 2016, 6. [CrossRef] [PubMed]

31. Ding, D.W.; Shu, C.J.; Sun, X. Cofactors Reconcile Multiple Signals in the Extracellular Electron Transfer Pathways in Shewanella oneidensis MR-1. J. Theor. Biol. 2017, under review.

32. Tatsuta, T.; Joo, D.M.; Calendar, R.; Akiyama, Y.; Ogura, T. Evidence for an active role of the DnaK chaperone system in the degradation of sigma 32. FEBS Lett. 2000, 478, 271-275. [CrossRef]

33. Callebaut, I.; Prat, K.; Meurice, E.; Mornon, J.P.; Tomavo, S. Prediction of the general transcription factors associated with RNA polymerase II in Plasmodium falciparum: Conserved features and differences relative to other eukaryotes. BMC Genom. 2005, 6. [CrossRef] [PubMed]

34. Agarwal, D.; Gregory, S.T.; O'Connor, M. Error-prone and error-restrictive mutations affecting ribosomal protein S12. J. Mol. Biol. 2011, 410, 1-9. [CrossRef] [PubMed] 
35. Mathy, N.; Pellegrini, O.; Serganov, A.; Patel, D.J.; Ehresmann, C.; Portier, C. Specific recognition of rpsO mRNA and 16S rRNA by Escherichia coli ribosomal protein S15 relies on both mimicry and site differentiation. Mol. Microbiol. 2004, 52, 661-675. [CrossRef] [PubMed]

36. Gao, H.; Barua, S.; Liang, Y.; Wu, L.; Dong, Y.; Reed, S.; Chen, J.; Culley, D.; Kennedy, D.; Yang, Y.; et al. Impacts of Shewanella oneidensis c-type cytochromes on aerobic and anaerobic respiration. Microbial. Biotechnol. 2010, 3, 455-466. [CrossRef] [PubMed]

37. Nelson, K.K.; Melendez, J.A. Mitochondrial redox control of matrix metalloproteinases. Free Radic. Biol. Med. 2004, 37, 768-784. [CrossRef] [PubMed]

38. Alon, U. Network motifs: Theory and experimental approaches. Nat. Rev. Genet. 2007, 8, 450-461. [CrossRef] [PubMed]

39. Ding, D.W.; Sun, X. A Comparative Study of Network Motifs in the Integrated Transcriptional Regulation and Protein Interaction Networks of Shewanella. IEEE/ACM Trans. Comput. Biol. Bioinform. 2017, under review.

40. Fischer, E.; Sauer, U. Large-scale in vivo flux analysis shows rigidity and suboptimal performance of Bacillus subtilis metabolism. Nat. Genet. 2005, 37, 636-640. [CrossRef] [PubMed]

41. Price, M.N.; Deutschbauer, A.M.; Skerker, J.M.; Wetmore, K.M.; Ruths, T.; Mar, J.S.; Kuehl, J.V.; Shao, W.; Arkin, A.P. Indirect and suboptimal control of gene expression is widespread in bacteria. Mol. Syst. Biol. 2013, 9, 660. [CrossRef] [PubMed]

42. Price, M.N.; Wetmore, K.M.; Deutschbauer, A.M.; Arkin, A.P. A Comparison of the Costs and Benefits of Bacterial Gene Expression. PLoS ONE 2016, 11, e0164314. [CrossRef] [PubMed]

43. Chung, S.S.; Pandini, A.; Annibale, A.; Coolen, A.C.; Thomas, N.S.; Fraternali, F. Bridging topological and functional information in protein interaction networks by short loops profiling. Sci. Rep. 2015, 5, 8540. [CrossRef] [PubMed]

44. Su, N.Y.; Ouni, I.; Papagiannis, C.V.; Kaiser, P. A dominant suppressor mutation of the met30 cell cycle defect suggests regulation of the Saccharomyces cerevisiae Met4-Cbf1 transcription complex by Met32. J. Biol. Chem. 2008, 283, 11615-11624. [CrossRef] [PubMed]

45. Shi, L.; Squier, T.C.; Zachara, J.M.; Fredrickson, J.K. Respiration of metal (hydr)oxides by Shewanella and Geobacter: A key role for multihaem c-type cytochromes. Mol. Microbiol. 2007, 65, 12-20. [CrossRef] [PubMed]

46. Hartshorne, R.S.; Reardon, C.L.; Ross, D.; Nuester, J.; Clarke, T.A.; Gates, A.J.; Mills, P.C.; Fredrickson, J.K.; Zachara, J.M.; Shi, L.; et al. Characterization of an electron conduit between bacteria and the extracellular environment. Proc. Natl. Acad. Sci. USA 2009, 106, 22169-22174. [CrossRef] [PubMed]

47. Coursolle, D.; Gralnick, J.A. Modularity of the Mtr respiratory pathway of Shewanella oneidensis strain MR-1. Mol. Microbiol. 2010, 77, 995-1008. [CrossRef] [PubMed]

48. Marritt, S.J.; Lowe, T.G.; Bye, J.; McMillan, D.G.; Shi, L.; Fredrickson, J.; Zachara, J.; Richardson, D.J.; Cheesman, M.R.; Jeuken, L.J.; et al. A functional description of CymA, an electron-transfer hub supporting anaerobic respiratory flexibility in Shewanella. Biochem. J. 2012, 444, 465-474. [CrossRef] [PubMed]

49. White, G.F.; Shi, Z.; Shi, L.; Wang, Z.; Dohnalkova, A.C.; Marshall, M.J.; Fredrickson, J.K.; Zachara, J.M.; Butt, J.N.; Richardson, D.J.; et al. Rapid electron exchange between surface-exposed bacterial cytochromes and Fe(III) minerals. Proc. Natl. Acad. Sci. USA 2013, 110, 6346-6351. [CrossRef] [PubMed]

50. Breuer, M.; Rosso, K.M.; Blumberger, J.; Butt, J.N. Multi-haem cytochromes in Shewanella oneidensis MR-1: Structures, functions and opportunities. J. R. Soc. Interface 2015, 12. [CrossRef] [PubMed]

51. Plate, L.; Marletta, M.A. Nitric oxide modulates bacterial biofilm formation through a multicomponent cyclic-di-GMP signaling network. Mol. Cell 2012, 46, 449-460. [CrossRef] [PubMed]

52. Maier, T.M.; Myers, C.R. The outer membrane protein Omp35 affects the reduction of Fe(III), nitrate, and fumarate by Shewanella oneidensis MR-1. BMC Microbiol. 2004, 4, 23. [CrossRef] [PubMed]

53. Szeinbaum, N.; Burns, J.L.; DiChristina, T.J. Electron transport and protein secretion pathways involved in Mn(III) reduction by Shewanella oneidensis. Environ. Microbiol. Rep. 2014, 6, 490-500. [CrossRef] [PubMed]

54. Kouzuma, A.; Oba, H.; Tajima, N.; Hashimoto, K.; Watanabe, K. Electrochemical selection and characterization of a high current-generating Shewanella oneidensis mutant with altered cell-surface morphology and biofilm-related gene expression. BMC Microbiol. 2014, 14, 190. [CrossRef] [PubMed]

55. Liu, T.; Yu, Y.Y.; Deng, X.P.; Ng, C.K.; Cao, B.; Wang, J.Y.; Rice, S.A.; Kjelleberg, S.; Song, H. Enhanced Shewanella biofilm promotes bioelectricity generation. Biotechnol. Bioeng. 2015, 112, 2051-2059. [CrossRef] [PubMed] 
56. Barnhart, M.M.; Chapman, M.R. Curli biogenesis and function. Annu. Rev. Microbiol. 2006, 60, $131-147$. [CrossRef] [PubMed]

57. Price, M.S.; Chao, L.Y.; Marletta, M.A. Shewanella oneidensis MR-1 H-NOX regulation of a histidine kinase by nitric oxide. Biochemistry 2007, 46, 13677-13683. [CrossRef] [PubMed]

58. Erbil, W.K.; Price, M.S.; Wemmer, D.E.; Marletta, M.A. A structural basis for H-NOX signaling in Shewanella oneidensis by trapping a histidine kinase inhibitory conformation. Proc. Natl. Acad. Sci. USA 2009, 106, 19753-19760. [CrossRef] [PubMed]

59. Fredrickson, J.K.; Romine, M.F.; Beliaev, A.S.; Auchtung, J.M.; Driscoll, M.E.; Gardner, T.S.; Nealson, K.H.; Osterman, A.L.; Pinchuk, G.; Reed, J.L.; et al. Towards environmental systems biology of Shewanella. Nat. Rev. Microbiol. 2008, 6, 592-603. [CrossRef] [PubMed]

60. Sundararajan, A.; Kurowski, J.; Yan, T.; Klingeman, D.M.; Joachimiak, M.P.; Zhou, J.; Naranjo, B.; Gralnick, J.A.; Fields, M.W. Shewanella oneidensis MR-1 Sensory Box Protein Involved in Aerobic and Anoxic Growth. Appl. Environ. Microbiol. 2011, 77, 4647-4656. [CrossRef] [PubMed]

61. Batagelj, V.; Mrvar, A. Pajek-Program for large network analysis. Connections 1998, 21, 47-57.

(C) 2018 by the authors. Licensee MDPI, Basel, Switzerland. This article is an open access article distributed under the terms and conditions of the Creative Commons Attribution (CC BY) license (http://creativecommons.org/licenses/by/4.0/). 